\title{
ONE-POT SYNTHESIS OF POLYSUBSTITUTED IMIDAZOLES FROM ARYLALDEHYDES IN WATER CATALYZED BY NHC USING MICROWAVE IRRADIATION
}

\author{
LEI WU ${ }^{1}$, XIAOBI JING ${ }^{11^{*}}$, HONGXIANG ZHU², YINLIN LIU ${ }^{1}$, CHAOGUO YAN \\ ${ }^{1}$ College of Chemistry and Chemical Engineering, Yangzhou University, Yangzhou 225002, P. R. China \\ ${ }^{2}$ School of Light Industrial and Food Engineering, Guangxi University, Nanning, P. R. China \\ (Received: January, 2011 - Accepted: January 26, 2012)
}

\begin{abstract}
A simple, high yielding synthesis of tri (3a-i) and tetrasubstituted (4a-g) imidazols from aldehydes is described. The cornerstone of this methodology involves the condensation of $\mathrm{NH}_{4} \mathrm{OAc}$, substituted aldehydes, and benzoin, which is synthesized in situ from aldehydes catalyzed by N-heterocyclic carbine (NHC), under microwave irradiation in water to afford trisubstituted imidazoles (3a-i). If arylamine is added in the solution, tetrasubstituted imidazoles (4a-g) can be obtained. Lepidilines B and trifenagrel are also synthesized in high yield using this procedure. All the experiment deta are in agreement with the literature.
\end{abstract}

Keywords: Trisubstituted imidazoles, tetrasubstituted imidazoles, microwave irradiation, Lepidilines B, Trifenagrel, N-heterocyclic carbine (NHC)

\section{INTRODUCTION}

Imidazole ring system is one of the most important substructures found in a large number of natural products and pharmacologically active compounds such as antiulcerative agent cimetidine ${ }^{1}$, the proton pump inhibitor omeprazole ${ }^{2}$ and the benzodiazepine antagonist flumazenil ${ }^{3}$ are imidazole derivatives. In addition, the substituted imidazole ring system is substantially used in ionic liquids ${ }^{4}$ that have been given a new approach to "green chemistry".

Triarylimidazole or tetraarylimidazole compounds have gained remarkable importance due to their widespread biological activities and their use in synthetic chemistry. ${ }^{5}$

In the literature, there are several methods reported for the synthesis of 2,4,5-triarylimidazoles using zeolite $\mathrm{HY} /$ silica gel ${ }^{6}, \mathrm{ZrCl}_{4}{ }^{7}, \mathrm{NiCl}_{2} \cdot 6 \mathrm{H}_{2} \mathrm{O}$, ionic liquid ${ }^{9}$, iodine ${ }^{10}$, Sodium bisulfite ${ }^{11}$ from the starting material of benzil. However, these methods require prolonged reaction time, exotic reaction condition and most importantly require expensive and hazardous acid or metal catalysts.

General methods relay on the synthesis of trisubstituted imidazoles followed by installation of the fourth substituent via N-alkylation ${ }^{[1]}$, metal activated coupling ${ }^{12}$, or imidazole-N-oxides ${ }^{13}$ to generate tetrasubstituted imidazoles.

Tetrasubstituted imidazoles can be directly prepared from benzil or benzoin condensation with aldehydes, amines and ammonium acetate but in this procedure the hazardous catalyst of $\mathrm{K}_{5} \mathrm{CoW}_{12} \mathrm{O}_{40} \cdot 3 \mathrm{H}_{2} \mathrm{O}{ }^{14}$ or molecular iodine ${ }^{15}$ is needed.

Wolkenberg ${ }^{15 b}$ reported in 2004 that 1,2-diketones and aldehydes can be condensed to form trisubstituted imidazoles in the presence of $\mathrm{NH}_{4} \mathrm{OAc}$ without any catalyst, but this method can only be used to synthesize trisubstituted imidazoles. Moreover, the synthesis of trisubstituted imidazoles was carried out in acetic acid leading to complex isolation and recovery procedures.

Therefore, the development of simple, efficient, inexpensive providing convenient procedure with improved yield for the synthesis of polysubstituted imidazoles is necessary.

To the best of our knowledge, one-pot synthesis of polysubstituted imidazoles directly from arylaldehyde in stead of benzil or benzoin has not been reported in the literature. Very recently, we reported ${ }^{16}$ our work on the synthesis of 1,2-diketones from arylaldehyde via organocatalysis. Based on this work, we developed a new method to synthesize polysubstituted imidazoles in one-pot from the starting material of arylaldehyde. Herein, we disclose our experimental result.

\section{RESULTS AND DISCUSSION}

Initially, we supposed triphenyl imidazole can be synthesized in onepot from the condensation of phenylaldehyde, $\mathrm{NH}_{4} \mathrm{OAc}$ and benzil, which was synthesized in situ according our previous method ${ }^{16}$. Experimental test resulted in very good yield, as shown in Scheme 1.

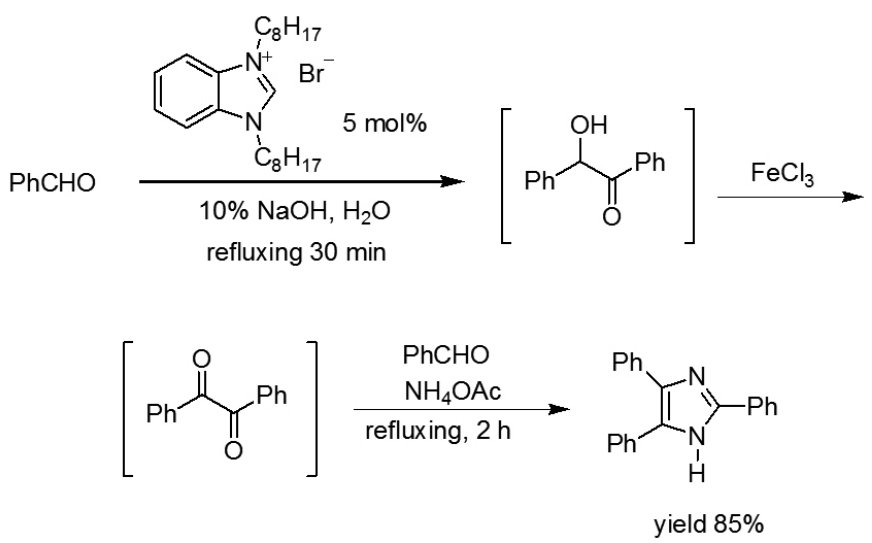

Scheme 1 synthesis of triphenyl imidazole

We have reported ${ }^{[16]}$ that if $\mathrm{FeCl}_{3}$ was not used after the condensation of aldehyde the mixture of benzil and benzoin can be obtained because of the air oxidation. Base on this discovery, we tried to obtain triphenyl imidazole in onepot without any oxydation of metal ion which is unfriendly to environment. Initial result shown that when aldehyde and $\mathrm{NH}_{4} \mathrm{OAc}$ were added directly into the benzil and benzoin mixture solution, which was obtained from aldehyde in situ catylized by N-heterocyclic carbene, after reflux for $12 \mathrm{~h}$ under air condition without any $\mathrm{FeCl}_{3}$, the yield of triphenyl imidazole was also very good, as shown in Scheme 2 .

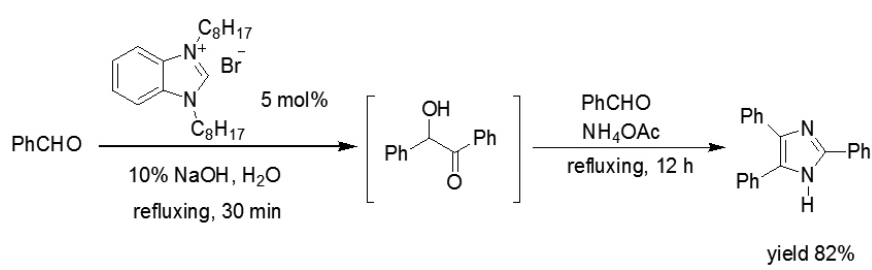

Scheme 2 synthesis of triphenyl imidazole without any metal oxidant

In order to deduce the reaction time, improved method, such as MAOS, was tested to be used in this reaction. It is well known that microwave-assisted organic synthesis (MAOS) has had a significant impact on synthetic chemistry. Reductions in reaction time, increase in yield and suppression of side product formation have all been described for microwave conditions relative to conventional thermal heating ${ }^{17,5 b}$. Based on this knowledge, microwave irradiation was used instead of refluxing in the one-pot sequence under air atmosphere. Impressively, the yield of triphenyl imidazole can be improved to $95 \%$ while the reaction time was deduced to only $10 \mathrm{~min}$. (Scheme 3 ) 


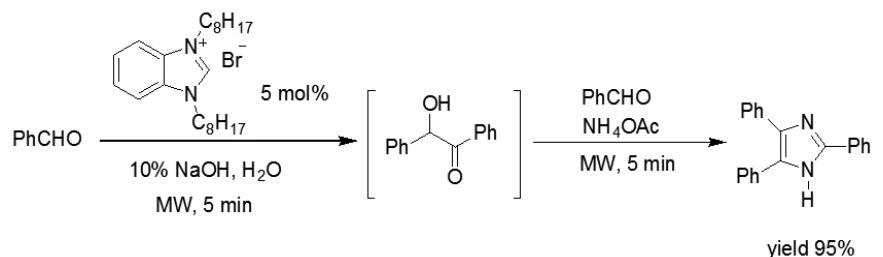

Scheme 3 synthesis of triphenyl imidazole using microwave irradiation

Different solvents were also screened in this reaction, resulting that water is the best solvent. All the results are summarized in Table 1.

Table 1. Synthesis of triphenyl imidazole in different solvents

\begin{tabular}{|c|c|c|c|}
\hline Entry & Solvent & Time(min) & ${\text { Yield }(\%)^{\mathrm{a}}}$ \\
\hline 1 & $\mathrm{CH}_{3} \mathrm{CH}_{2} \mathrm{OH}$ & $5+5$ & 87 \\
2 & $\mathrm{THF}$ & $5+5$ & 66 \\
3 & $\mathrm{H}_{2} \mathrm{O}$ & $5+5$ & 95 \\
4 & $\mathrm{CH}_{3} \mathrm{CN}$ & $5+5$ & 65 \\
\hline
\end{tabular}

a: isolated yields

Encouraged by the aforementioned results and with the suitable reaction conditions in hand, we tested the feasibility of the protocol using various arylaldehydes. Fortunately, this procedure provided a straight forward synthetic route to the trisubstituted imidazoles. All the results are list in Scheme 4 and Table 2.

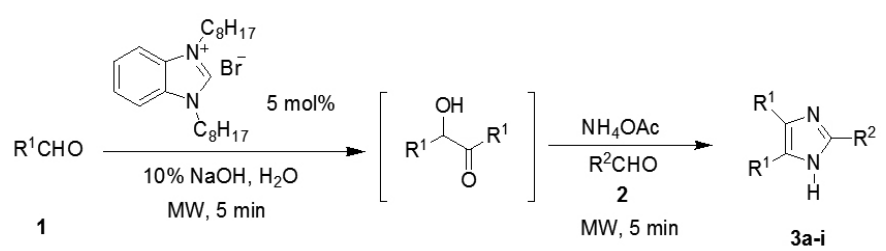

Scheme 4 synthesis of trisubsituted imidazoles.

Table 2. one-pot synthesis of trisubstituted imidazoles from different arylaldehydes.

\begin{tabular}{|c|c|c|c|c|c|}
\hline Entry & $\mathrm{R}^{1}$ & $\mathrm{R}^{2}$ & Product & $\underset{\mathrm{a}}{\text { Yield(\%) }}$ & $\mathrm{Mp}\left({ }^{\circ} \mathrm{C}\right)$ \\
\hline 1 & $\mathrm{Ph}$ & $\mathrm{Ph}$ & $3 a$ & 95 & $276-278$ \\
\hline 2 & $\mathrm{Ph}$ & $4-\mathrm{CH}_{3}-\mathrm{ph}$ & $3 b$ & 94 & $231-232$ \\
\hline 3 & 4- $\mathrm{CH}_{3} \mathrm{O}-\mathrm{ph}$ & 4- $\mathrm{CH}_{3} \mathrm{O}-\mathrm{ph}$ & $3 c$ & 82 & $256-257$ \\
\hline 4 & 3-Cl-ph & $\mathrm{Ph}$ & $3 \mathrm{~d}$ & 96 & $282-284$ \\
\hline 5 & 3-Cl-ph & 4- $\mathrm{CH}_{3} \mathrm{O}-\mathrm{ph}$ & $3 e$ & 94 & $238-240$ \\
\hline 6 & 3-Br-ph & $\mathrm{Ph}$ & $3 f$ & 96 & $272-274$ \\
\hline 7 & 4-Cl-ph & $\mathrm{Ph}$ & $3 \mathrm{~g}$ & 98 & 296-298 \\
\hline 8 & 4-Cl-ph & 2-OH-ph & $3 \mathrm{~h}$ & 92 & $236-238$ \\
\hline 9 & $4-\mathrm{CH}_{3}-\mathrm{ph}$ & $4-\mathrm{CH}_{3}-\mathrm{ph}$ & $3 \mathrm{i}$ & 85 & $240-242$ \\
\hline 10 & 2-Furanyl & $\mathrm{Ph}$ & $3 \mathrm{j}$ & trace & --- \\
\hline 11 & 2-Pyridinyl & $3-\mathrm{NO}_{2}-\mathrm{ph}$ & $3 \mathrm{k}$ & trace & --- \\
\hline 12 & $3-\mathrm{NO}_{2}-\mathrm{ph}$ & $\mathrm{Ph}^{2}$ & 31 & none & --- \\
\hline 13 & $4-\mathrm{NO}_{2}-\mathrm{ph}$ & $4-\mathrm{CH}_{3}$ & $3 \mathrm{~m}$ & none & --- \\
\hline 14 & 4-OH-ph & 4-Cl-ph & $3 n$ & none & --- \\
\hline
\end{tabular}

a: isolated yields

From table 2, it shows that the yields of trisubstituted imidazoles from the starting material of phenyl aldehyde bearing chloro, bromo or methyl substitute were very good, while using furanyl, pyridinyl, nitro and hydroxyl phenyl aldehyde, trisubstituted imidazoles can not be obtained, because these kinds of benzoins can not be synthesized in situ.

When arylamine along with $\mathrm{NH}_{4} \mathrm{OAc}$ and arylaldehyde were added into the benzil and benzoin mixture solution, under the same condition discribed above, tetrasubstituted imidazoles could also be synthesized. All the results are list in scheme 5 and table 3 .

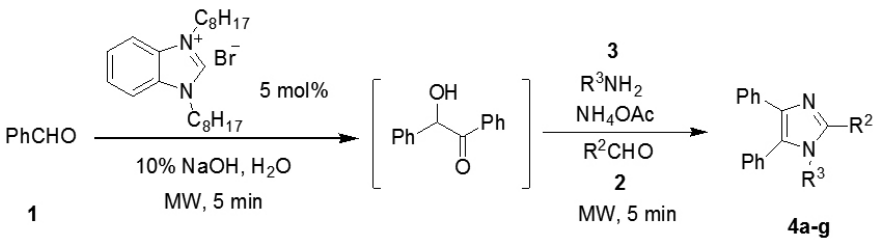

Scheme 5 synthesis of terisubsituted imidazoles

Table 3. One-pot synthesis of tetrasubstituted imidazoles from different arylamines

\begin{tabular}{|c|c|c|c|c|c|}
\hline Entry & $\mathrm{R}^{2}$ & $\mathrm{R}^{3}$ & Product & Yield $(\%)^{\mathrm{a}}$ & $\mathrm{Mp}\left({ }^{\circ} \mathrm{C}\right)$ \\
\hline 1 & $\mathrm{Ph}$ & $\mathrm{Ph}$ & $4 \mathrm{a}$ & 91 & $163-165$ \\
2 & $\mathrm{Ph}$ & 4-Cl-ph & $4 \mathrm{~b}$ & 87 & $192-194$ \\
3 & $4-\mathrm{CH}_{3} \mathrm{O}-\mathrm{ph}$ & 4- $\mathrm{CH}_{3}-\mathrm{ph}$ & $4 \mathrm{c}$ & 89 & $180-182$ \\
4 & $4-\mathrm{CH}_{3}-\mathrm{ph}$ & $\mathrm{Ph}^{-}$ & $4 \mathrm{~d}$ & 88 & $155-157$ \\
5 & $\mathrm{Ph}^{-}$ & 4- $\mathrm{CH}_{3}-\mathrm{ph}$ & $4 \mathrm{e}$ & 88 & $172-174$ \\
6 & $4-\mathrm{CH}_{3}-\mathrm{ph}$ & 4-Cl-ph & $4 \mathrm{f}$ & 86 & $167-169$ \\
7 & $\mathrm{Ph}$ & 3-Cl-ph & $4 \mathrm{~g}$ & 84 & $144-146$ \\
\hline
\end{tabular}

a: isolated yields

Lepidiline $\mathrm{B}^{18}$, which exhibit micromolar cytotoxicity against several human cancer cell lines, were isolated from the root extract of lepidium meyenii collected from the Andes Mountains of Peru during a search for bioactive natural products. We synthesized Lepidiline B using our method described above. (Scheme 6) Lepidiline B was first synthesized in a two-step procedure from 2, 3-butanedione and acetaldehyde in $43 \%$ overall yield. While using our one-pot procedure directly from acetaldehyde, the yield is about $67 \%$.

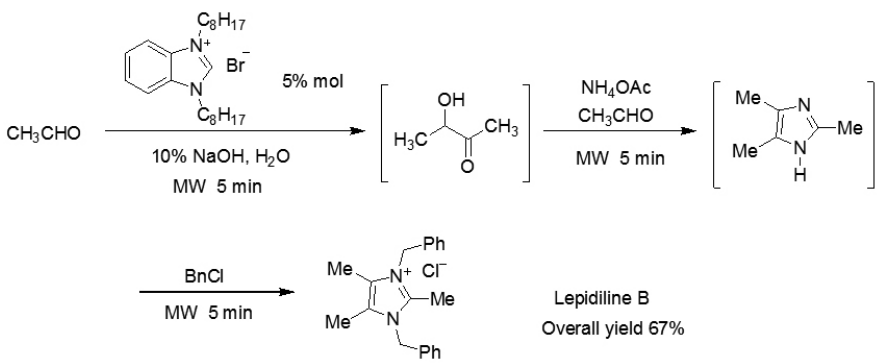

Scheme 6 synthesis of Lepidiline B

Trifenagrel ${ }^{19}$ is a potent arachidonate cyclo-oxygenase inhibitor that reduces platelet aggregation in several animal species and humans. Preparation of the drug using our one-pot condensation reaction proceeded smoothly and in high yield. This example highlights the speed of the method: whereas the existing optimized procedure for its preparation furnishes product after $2 \mathrm{~h}$ at reflux from dione, the one-pot protocol delivers trifenagrel in $95 \%$ yield after 10 min directly from aldehyde. (Scheme 7)

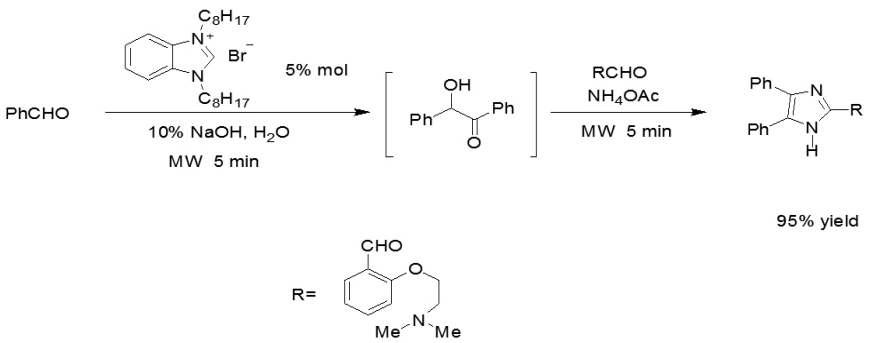

Scheme 7 synthesis of trifenagrel 
In conclusion, we have reported here in several noteworthy features of a new method for the synthesis of polysubstituted imidazoles from aldehydes in one-pot using microwave irradiation. This protocol offers many attractive features such as reduced reaction times, higher yields and economic viability of the one-pot sequence.

\section{EXPERIMENTAL}

\section{General}

Melting points were obtained on a hot-plate microscope apparatus and were uncorrected. ${ }^{1} \mathrm{H}$ and ${ }^{13} \mathrm{C}$ NMR spectra were recorded with a Bruker AV600 spectrophotometer. IR spectra were obtained on a Nicolet FT-IR 740 spectrometer $(\mathrm{KBr}$ disc). X-ray data were collected on a Bruker Smart APEX-2 diffractometer.

General procedure for the synthesis of triarylimidazole (3a-i)

A mixture of benzimidazolium salt $(0.25 \mathrm{mmol}, 0.11 \mathrm{~g})$, arylaldehyde $(5 \mathrm{mmol}), 2 \mathrm{~mL} 10 \%$ aqua $\mathrm{NaOH}$ and $10 \mathrm{~mL}$ water was irradiated at $350 \mathrm{w}$ for $5 \mathrm{~min}$ in a microwave synthesizer. After adding HOAc to $\mathrm{pH}=6-7$, then $\mathrm{NH}_{4} \mathrm{OAc}(10 \mathrm{mmol}, 0.77 \mathrm{~g})$ and aldehyde $(5 \mathrm{mmol})$ were added. The mixture was irradiated for $5 \mathrm{~min}$ again. The reaction mixture was allowed to cool to 0 ${ }^{\circ} \mathrm{C}$. The precipitated solid was filtered. The crude product on recrystallization from $\mathrm{EtOH}$ yielded the requisite trisubstituted imidazoles.

\section{General procedure for the synthesis of tetraarylimidazole (4a-g)}

A mixture of benzimidazolium salt $(0.25 \mathrm{mmol}, 0.11 \mathrm{~g})$, arylaldehyde (5 $\mathrm{mmol}$ ), $2 \mathrm{~mL} 10 \%$ aqua $\mathrm{NaOH}$ and $10 \mathrm{~mL}$ water was irradiated at $350 \mathrm{w}$ for 5 min in a microwave synthesizer. After adding $\mathrm{HOAc}$ to $\mathrm{pH}=6-7$, then $\mathrm{NH}_{4} \mathrm{OAc}$ $(5 \mathrm{mmol}, 0.39 \mathrm{~g})$, aldehyde $(5 \mathrm{mmol})$ and arylamine $(5 \mathrm{mmol})$ were added. The mixture was irradiated for 5 min again. The reaction mixture was allowed to cool to $0{ }^{\circ} \mathrm{C}$. The precipitated solid was filtered. The crude product on recrystallization from EtOH yielded the requisite tetrasubstituted imidazoles.

\section{The synthesis of Lepidiline $B$}

A mixture of benzimidazolium salt $(0.25 \mathrm{mmol}, 0.11 \mathrm{~g})$, Acetaldehyde $(5$ mmol, $0.22 \mathrm{~g}$ ), $2 \mathrm{~mL} 10 \%$ aqua $\mathrm{NaOH}$ and $10 \mathrm{~mL}$ water was irradiated at $350 \mathrm{w}$ for $5 \mathrm{~min}$ in a microwave synthesizer. After adding $\mathrm{HOAc}$ to $\mathrm{pH}=6-7, \mathrm{NH}_{4} \mathrm{OAc}$ $(10 \mathrm{mmol}, 0.77 \mathrm{~g})$ and Acetaldehyde $(5 \mathrm{mmol}, 0.22 \mathrm{~g})$ were added. The mixture was irradiated for $5 \mathrm{~min}$ again. The reaction mixture was allowed to cool to room temperature. Then Benzyl chloride $(1.27 \mathrm{~g}, 10 \mathrm{mmol})$ and $1.5 \mathrm{~mL} \mathrm{Et}_{3} \mathrm{~N}$ were added. The mixture was continually irradiated for $5 \mathrm{~min}$. The reaction mixture was allowed to cool to $0{ }^{\circ} \mathrm{C}$. The precipitated solid was filtered. The crude product on recrystallization from $\mathrm{EtOH}$ yielded the requisite natural product Lepidiline B $(1.089 \mathrm{~g}, 67 \%)$ as a solid, mp: $220-222{ }^{\circ} \mathrm{C} ;{ }^{1} \mathrm{H}$ NMR $(600$ MHz, DMSO- $d$ ) $\delta: 7.45-7.36(\mathrm{~m}, 6 \mathrm{H}, \mathrm{Ar}-\mathrm{H}), 7.15(\mathrm{~d}, J=7.2 \mathrm{~Hz}, 4 \mathrm{H}, \mathrm{Ar}-\mathrm{H})$, $5.45\left(\mathrm{~s}, 4 \mathrm{H}, \mathrm{CH}_{2}\right), 2.63\left(\mathrm{~s}, 3 \mathrm{H}, \mathrm{CH}_{3}\right), 2.13\left(\mathrm{~s}, 6 \mathrm{H}, \mathrm{CH}_{3}\right) ;{ }^{13} \mathrm{CNMR}(150 \mathrm{MHz}$, DMSO- $d$ ) $\delta: 143.6(\mathrm{C}=\mathrm{N}), 134.5,129.0,128.3,126.5,125.8,47.7\left(\mathrm{CH}_{2}\right), 10.3$ $\left(\mathrm{CH}_{3}\right), 8.2\left(\mathrm{CH}_{3}\right)$; IR $(\mathrm{KBr}): 3400\left(\mathrm{H}_{2} \mathrm{O}\right), 2360\left(\mathrm{~N}^{+}=\mathrm{C}\right), 1648(\mathrm{C}=\mathrm{C}), 1525(\mathrm{Ph})$, $1454(\mathrm{Ph}), 742(\mathrm{Ph}), 712(\mathrm{Ph}) \mathrm{cm}^{-1}$.

\section{The synthesis of Trifenagrel}

A mixture of benzimidazolium salt $(0.25 \mathrm{mmol}, 0.11 \mathrm{~g})$, benzaldehyde $(5$ mmol, $0.53 \mathrm{~g}$ ), $2 \mathrm{~mL} 10 \%$ aqua $\mathrm{NaOH}$ and $10 \mathrm{~mL}$ water was irradiated at $350 \mathrm{w}$ for $5 \mathrm{~min}$ in a microwave synthesizer. After adding HOAc to $\mathrm{pH}=6-7$, then $\mathrm{NH}_{4} \mathrm{OAc}(10 \mathrm{mmol}, 0.77 \mathrm{~g})$ and 2-[2-(dimethylamino)ethoxy]benzaldehyde $(5 \mathrm{mmol}, 0.98 \mathrm{~g}$ ) were added. The mixture was irradiated for $5 \mathrm{~min}$ again. The reaction mixture was allowed to cool to $0{ }^{\circ} \mathrm{C}$. The precipitated solid was filtered. The crude product on recrystallization from $\mathrm{EtOH}$ yielded the requisite natural product Trifenagrel $(1.819,95 \%)$ as a solid, mp: $147-148^{\circ} \mathrm{C} ;{ }^{1} \mathrm{H}$ NMR $\left(600 \mathrm{MHz}, \mathrm{CDCl}_{3}\right) \delta: 12.20(\mathrm{~s}, 1 \mathrm{H}, \mathrm{N}-\mathrm{H}), 8.48$ (d, $\left.J=7.8 \mathrm{~Hz}, 1 \mathrm{H}, \mathrm{Ar}-\mathrm{H}\right), 7.64$ $(\mathrm{d}, J=7.2 \mathrm{~Hz}, 2 \mathrm{H}, \mathrm{Ar}-\mathrm{H}), 7.48(\mathrm{~d}, J=6.3 \mathrm{~Hz}, 2 \mathrm{H}, \mathrm{Ar}-\mathrm{H}), 7.40-7.27(\mathrm{~m}, 8 \mathrm{H}$, Ar-H), $7.11(\mathrm{t}, J=7.5 \mathrm{~Hz}, 1 \mathrm{H}, \mathrm{Ar}-\mathrm{H}), 7.01(\mathrm{~d}, J=8.4 \mathrm{~Hz}, 1 \mathrm{H}, \mathrm{Ar}-\mathrm{H}), 4.24$ (dd, $\left.J=5.1,6.0 \mathrm{~Hz}, 2 \mathrm{H}, \mathrm{CH}_{2}-\mathrm{O}\right), 2.67\left(\mathrm{dd}, J=5.1,5.4 \mathrm{~Hz}, 2 \mathrm{H}, \mathrm{CH}_{2}-\mathrm{N}\right), 1.98(\mathrm{~s}, 6 \mathrm{H}$, $\left.\mathrm{CH}_{3}\right):{ }^{13} \mathrm{CNMR}\left(150 \mathrm{MHz}, \mathrm{CDCl}_{3}\right) \delta: 154.5(\mathrm{Ar}-\mathrm{O}), 146.9(\mathrm{C}=\mathrm{N}), 133.2,129.5$, 129.3, 128.6, 128.1, 127.1, 121.0, 117.2, 114.6, $66.8\left(\mathrm{CH}_{2}-\mathrm{O}\right), 58.9\left(\mathrm{CH}_{2}-\mathrm{N}\right)$, $45.5\left(\mathrm{CH}_{3}\right)$; IR (KBr): $3454(\mathrm{NH}), 3032(\mathrm{Ar}-\mathrm{H}), 1598(\mathrm{C}=\mathrm{N}), 1500(\mathrm{Ph}), 1254(\mathrm{C}-$ O), $1092(\mathrm{C}-\mathrm{O}-\mathrm{C}), 830(\mathrm{Ph}) \mathrm{cm}^{-1}$

\section{Spectral data for novel compounds:}

2,4,5-tris(4-methoxyphenyl)-1H-imidazole (3c): white solid; mp: 256-257 ${ }^{\circ} \mathrm{C}$; ${ }^{1} \mathrm{H}$ NMR $\left(600 \mathrm{MHz}, \mathrm{CDCl}_{3}\right) \delta: 7.91(\mathrm{~d}, J=8.3 \mathrm{~Hz}, 2 \mathrm{H}, \mathrm{Ar}-\mathrm{H}), 7.38(\mathrm{~d}, J$ $=8.4 \mathrm{~Hz}, 2 \mathrm{H}, \mathrm{Ar}-\mathrm{H}), 6.83(\mathrm{~d}, J=8.5 \mathrm{~Hz}, 6 \mathrm{H}, \mathrm{Ar}-\mathrm{H}), 3.82\left(\mathrm{~s}, 3 \mathrm{H}, \mathrm{OCH}_{3}\right), 3.81$ $\left(\mathrm{s}, 6 \mathrm{H}, \mathrm{OCH}_{3}\right) ;{ }^{13} \mathrm{C}$ NMR $\left(150 \mathrm{MHz}, \mathrm{CDCl}_{3}\right) \delta: 160.4$ (Ar-O), 159.2 (Ar-O), $144.9(\mathrm{C}=\mathrm{N}), 129.3,127.5,123.4,114.2,113.9,55.3(\mathrm{OCH}), 55.2(\mathrm{OCH}) ; \mathrm{IR}$ $(\mathrm{KBr}): 3450(\mathrm{NH}), 1611(\mathrm{C}=\mathrm{C}), 1501(\mathrm{Ph}), 1253(\mathrm{Ar}-\mathrm{H}), 1185(\mathrm{Ar}-\mathrm{H}), 1030(\mathrm{Ar}-$ $\mathrm{H}), 840(\mathrm{Ar}-\mathrm{H}) \mathrm{cm}^{-1}$.

4,5-bis(3-chlorophenyl)-2-phenyl-1H-imidazole (3d): white solid, mp: $282-284{ }^{\circ} \mathrm{C} ;{ }^{1} \mathrm{H}$ NMR $\left(600 \mathrm{MHz}, \mathrm{DMSO}-d_{6}\right) \delta: 12.83(\mathrm{~s}, 1 \mathrm{H}, \mathrm{N}-\mathrm{H}), 8.08(\mathrm{~d}, J$
$=7.3 \mathrm{~Hz}, 2 \mathrm{H}, \mathrm{Ar}-\mathrm{H}), 7.62(\mathrm{~d}, J=7.8 \mathrm{~Hz}, 2 \mathrm{H}, \mathrm{Ar}-\mathrm{H}), 7.51-7.43(\mathrm{~m}, 6 \mathrm{H}, \mathrm{Ar}-\mathrm{H})$, $7.41(\mathrm{t}, J=7.4 \mathrm{~Hz}, 1 \mathrm{H}, \mathrm{Ar}-\mathrm{H}), 7.35(\mathrm{t}, J=7.8 \mathrm{~Hz}, 1 \mathrm{H}, \mathrm{Ar}-\mathrm{H}), 7.31(\mathrm{~d}, J=8.2$ $\mathrm{Hz}, 1 \mathrm{H}, \mathrm{Ar}-\mathrm{H}) ;{ }^{13} \mathrm{C}$ NMR $\left(150 \mathrm{MHz}, \mathrm{DMSO}-d_{6}\right) \delta: 146.2(\mathrm{C}=\mathrm{N}), 133.4,133.0$, $132.6,130.6,130.2,129.9,128.7,128.6,127.9,127.8,127.4,127.0,126.6$, 126.5, 125.4, 125.3; IR (KBr): 3450(NH), $1600(\mathrm{C}=\mathrm{C}), 1585(\mathrm{Ph}), 889(\mathrm{Ar}-\mathrm{H})$, 788(Ar-H), 751(Ar-H), 690(Ar-H) $\mathrm{cm}^{-1}$.

4,5-bis(3-chlorophenyl)-2-(4-methoxyphenyl)-1H-imidazole (3e): white solid, mp: $238-240{ }^{\circ} \mathrm{C} ;{ }^{1} \mathrm{H}$ NMR $(600 \mathrm{MHz}$, DMSO- $d$ ) $\delta: 12.68(\mathrm{~s}, 1 \mathrm{H}$, $\mathrm{N}-\mathrm{H}), 8.02(\mathrm{~d}, J=8.7 \mathrm{~Hz}, 2 \mathrm{H}, \operatorname{Ar}-\mathrm{H}), 7.61(\mathrm{~s}, 2 \mathrm{H}, \mathrm{Ar}-\mathrm{H}), 7.44-7.31(\mathrm{~m}, 6 \mathrm{H}$, Ar-H), $7.06(\mathrm{~d}, J=8.8 \mathrm{~Hz}, 2 \mathrm{H}, \mathrm{Ar}-\mathrm{H}), 3.82\left(\mathrm{~s}, 3 \mathrm{H}, \mathrm{OCH}_{3}\right) ;{ }^{13} \mathrm{C}$ NMR $(150$ MHz, DMSO- $d$ ) $\delta: 159.7$ (Ar-O), $146.3(\mathrm{C}=\mathrm{N}), 133.4,130.5,130.1,127.8$, $126.9,125.5,122.6,114.1,55.20\left(\mathrm{OCH}_{3}\right)$; IR ( $\left.\mathrm{KBr}\right): 3452(\mathrm{NH}), 3027(\mathrm{Ar}-\mathrm{H})$, $1598(\mathrm{C}=\mathrm{C}), 1564(\mathrm{Ph}), 1249(\mathrm{Ar}-\mathrm{H}), 788(\mathrm{Ar}-\mathrm{H}) \mathrm{cm}^{-1}$

4,5-bis(3-bromophenyl)-2-phenyl-1H-imidazole (3f): white solid, mp: $272-274{ }^{\circ} \mathrm{C} ;{ }^{1} \mathrm{H}$ NMR $\left(600 \mathrm{MHz}, \mathrm{DMSO}-d_{\text {f }}\right) \delta: 12.87(\mathrm{~s}, 1 \mathrm{H}, \mathrm{N}-\mathrm{H}), 8.08(\mathrm{~d}, J$ $=7.8 \mathrm{~Hz}, 2 \mathrm{H}, \mathrm{Ar}-\mathrm{H}), 7.77(\mathrm{~s}, 2 \mathrm{H}, \mathrm{Ar}-\mathrm{H}), 7.61(\mathrm{~d}, J=6.9 \mathrm{~Hz}, 1 \mathrm{H}, \mathrm{Ar}-\mathrm{H}), 7.51-$ $7.45(\mathrm{~m}, 5 \mathrm{H}, \mathrm{Ar}-\mathrm{H}), 7.41(\mathrm{t}, J=7.3 \mathrm{~Hz}, 2 \mathrm{H}, \mathrm{Ar}-\mathrm{H}), 7.29(\mathrm{t}, J=7.5 \mathrm{~Hz}, 1 \mathrm{H}$, Ar-H); ${ }^{13} \mathrm{C}$ NMR (150 MHz, DMSO- $\left.d_{6}\right) \delta: 146.2(\mathrm{C}=\mathrm{N}), 135.3,133.1,131.7$, 131.5, 131.1, 130.7, 130.5, 129.8, 128.7, 128.6, 127.5, 125.3, 123.6; IR (KBr): $3417(\mathrm{NH}), 3060(\mathrm{Ar}-\mathrm{H}), 1588(\mathrm{C}=\mathrm{C}), 1466(\mathrm{Ph}), 1407(\mathrm{Ph}), 888(\mathrm{Ar}-\mathrm{H}), 784(\mathrm{Ar}-$ $\mathrm{H}), 691(\mathrm{Ar}-\mathrm{H}) \mathrm{cm}^{-1}$.

4,5-bis(4-chlorophenyl)-2-phenyl-1H-imidazole (3g): white solid, $\mathrm{mp:}$ 296-298 ${ }^{\circ} \mathrm{C} ;{ }^{1} \mathrm{H}$ NMR $\left(600 \mathrm{MHz}\right.$, DMSO- $\left.d_{6}\right) \delta: 12.76(\mathrm{~s}, 1 \mathrm{H}, \mathrm{N}-\mathrm{H}), 8.07$ (d, $J$ $=7.8 \mathrm{~Hz}, 2 \mathrm{H}, \mathrm{Ar}-\mathrm{H}), 7.55-7.47(\mathrm{~m}, 8 \mathrm{H}, \mathrm{Ar}-\mathrm{H}), 7.39$ (t, $J=7.4 \mathrm{~Hz}, 3 \mathrm{H}, \mathrm{Ar}-\mathrm{H})$; ${ }^{13} \mathrm{C}$ NMR $\left(150 \mathrm{MHz}\right.$, DMSO- $\left.d_{6}\right) \delta: 145.9(\mathrm{C}=\mathrm{N}), 133.7,132.4,131.1,130.1$, $130.0,129.5,128.8,128.7,128.4,128.3,127.3,125.2$; IR (KBr): $3446(\mathrm{NH})$, 1636(C=C), 1491( Ph), 1093(Ar-H), 833(Ar-H) $\mathrm{cm}^{-1}$.

2-(4,5-bis(4-chlorophenyl)-1H-imidazole-2-yl)phenol (3h): white solid, mp: $236-238^{\circ} \mathrm{C}$; ${ }^{1} \mathrm{H}$ NMR $\left(600 \mathrm{MHz}, \mathrm{DMSO}-d_{f}\right) \delta: 13.07$ (s, $\left.1 \mathrm{H}, \mathrm{N}-\mathrm{H}\right), 12.67$ (s, 1H), 8.02 (d, $J=7.8 \mathrm{~Hz}, 1 \mathrm{H}, \mathrm{Ar}-\mathrm{H}), 7.54-7.44$ (m, 8H, Ar-H), 7.29 (t, $J=$ 7.7 Hz, 1H, Ar-H), 6.99-6.95 (m, 2H, Ar-H); ${ }^{13} \mathrm{C}$ NMR (150 MHz, DMSO- $\left.d_{6}\right)$ $\delta: 156.5(\mathrm{Ar}-\mathrm{O}), 146.2(\mathrm{C}=\mathrm{N}), 130.4,130.3,128.9,128.7,128.5,125.0$, 118.9, 116.8, 112.6; IR (KBr): 3445(NH), 3235(C-H), $1590(\mathrm{C}=\mathrm{C}), 1495(\mathrm{Ph})$, 1430(Ph), 1254(Ar-H), 1093(Ar-H), 830(Ar-H) $\mathrm{cm}^{-1}$. Compound 3h were also confirmed by X-ray single crystal analysis as shown in Figure 1.

Figure 1. X-ray structure of compound $3 h$.

2,4,5-tri-p-tolyl-1H-imidazole (3i): white solid, mp: $240-242{ }^{\circ} \mathrm{C} ;{ }^{1} \mathrm{H}$ NMR $\left(600 \mathrm{MHz}, \mathrm{CDCl}_{3}\right) \delta: 9.60(\mathrm{~s}, 1 \mathrm{H}, \mathrm{N}-\mathrm{H}), 7.74$ (d, $\left.J=7.6 \mathrm{~Hz}, 2 \mathrm{H}, \mathrm{Ar}-\mathrm{H}\right), 7.40$ $(\mathrm{m}, 4 \mathrm{H}, \mathrm{Ar}-\mathrm{H}), 7.19(\mathrm{~d}, J=7.6 \mathrm{~Hz}, 2 \mathrm{H}, \mathrm{Ar}-\mathrm{H}), 7.11(\mathrm{~d}, J=6.9 \mathrm{~Hz}, 4 \mathrm{H}, \mathrm{Ar}-\mathrm{H})$, $2.36\left(\mathrm{~s}, 3 \mathrm{H}, \mathrm{CH}_{3}\right), 2.34\left(\mathrm{~s}, 6 \mathrm{H}, \mathrm{CH}_{3}\right) ;{ }^{13} \mathrm{C} \mathrm{NMR}\left(150 \mathrm{MHz}, \mathrm{CDCl}_{3}\right) \delta: 147.7$, $145.8(\mathrm{C}=\mathrm{N}), 138.6,138.3,135.8,129.6,129.5,129.2,128.9,127.6,127.2$, 125.1, 21.3 $\left(\mathrm{CH}_{3}\right), 21.2\left(\mathrm{CH}_{3}\right)$; IR $(\mathrm{KBr}): 3450(\mathrm{NH}), 3030(\mathrm{Ar}-\mathrm{H}), 1620(\mathrm{C}=\mathrm{C})$, 1499(Ph), 819(Ph-H), 727(Ph-H) $\mathrm{cm}^{-1}$.

2,4,5-triphenyl-1-(4-chlorophenyl)-imidazole (4b): white solid, mp: $192-$ $194{ }^{\circ} \mathrm{C} ;{ }^{1} \mathrm{H}$ NMR $(600 \mathrm{MHz}$, DMSO- $d$ ) $\delta: 7.45-7.57$ (m, 12H, Ar-H), 7.30(d $J=7.8 \mathrm{~Hz}, 2 \mathrm{H}, \mathrm{Ar}-\mathrm{H}), 7.22(\mathrm{~d} J=7.8 \mathrm{~Hz}, 2 \mathrm{H}, \mathrm{Ar}-\mathrm{H}), 7.40$ (m, 3H, Ar-H); IR $(\mathrm{KBr})$ : 3010(Ar-H), 1650(C=C), 1480(Ph), 800(Ph-H), $\mathrm{cm}^{-1}$

1-p-tolyl-2-(4-methoxylphenyl)-4,5-diphenylimidazole(4c): white solid, mp: $180-182^{\circ} \mathrm{C} .{ }^{1} \mathrm{H}$ NMR $(600 \mathrm{MHz}$, DMSO- $d$ ) $\delta: 7.74(\mathrm{~d}, J=7.6 \mathrm{~Hz}, 2 \mathrm{H}, \mathrm{Ar}-$ H), 7.42-7.50(m, 12h, Ar-H), 7.19 (d, $J=7.6 \mathrm{~Hz}, 2 \mathrm{H}, \mathrm{Ar}-\mathrm{H}), 7.05(\mathrm{~m}, 2 \mathrm{~h}, \mathrm{Ar}-$ $\mathrm{H}), 3.80\left(\mathrm{~s}, 3 \mathrm{H}, \mathrm{OCH}_{3}\right), 2.40\left(\mathrm{~s}, 3 \mathrm{H}, \mathrm{CH}_{3}\right)$, IR $(\mathrm{KBr}): 3003(\mathrm{C}-\mathrm{H}), 1600(\mathrm{C}=\mathrm{C})$, 1477( (Ph), 1428( (Ph), 1254(Ar-H), 1093(Ar-O), 830(Ar-H) cm-1.

1,4,5-triphenyl-2-p-tolylimidazole(4d): white solid, mp: $155-157{ }^{\circ} \mathrm{C} .{ }^{1} \mathrm{H}$ NMR (600 MHz, DMSO- $d$ ) $\delta: 7.80$ (d, $J=7.4 \mathrm{~Hz}, 2 \mathrm{H}, \mathrm{Ar}-\mathrm{H}), 7.53$ (d, $J=7.4$ $\mathrm{Hz}, 2 \mathrm{H}, \mathrm{Ar}-\mathrm{H}), 7.38-7.40(\mathrm{~m}, 12 \mathrm{~h}, \mathrm{Ar}-\mathrm{H}), 7.13(\mathrm{~m}, 3 \mathrm{~h}, \mathrm{Ar}-\mathrm{H}), 2.35$ (s, 3H, $\left.\mathrm{CH}_{3}\right)$, IR (KBr): 2999(C-H), 1593(C=C), 1460(Ph), 1255(Ar-H), 833(Ar-H) $\mathrm{cm}^{-1}$.

2,4,5-triphenyl-1-p-tolylimidazole (4e) white solid, mp: $172-174{ }^{\circ} \mathrm{C} .{ }^{1} \mathrm{H}$ NMR (600 MHz, DMSO- $d$ ) $\delta: 7.43-7-55(\mathrm{~m}, 12 \mathrm{H}, \mathrm{Ar}-\mathrm{H}), 7.30-7.39(2 \mathrm{~m}, 12 \mathrm{~h}$, Ar-H), 7.26 (d, J=7.4 Hz, 2H, Ar-H), 7.13(m, 3h, Ar-H), 2.35 (s, 3H, $\left.\mathrm{CH}_{3}\right)$, IR $(\mathrm{KBr}): 3090(\mathrm{C}-\mathrm{H}), 1515(\mathrm{C}=\mathrm{C}), 1500(\mathrm{Ph}), 1234(\mathrm{Ar}-\mathrm{H}), 775(\mathrm{Ar}-\mathrm{H}) \mathrm{cm}^{-1}$.

1-p-chlorophenyl-2-p-tolyl-4,5-diphenylimdazole(4f) white solid, mp: $167-169^{\circ} \mathrm{C} .{ }^{1} \mathrm{H}$ NMR $(600 \mathrm{MHz}$, DMSO- $d$ ) $\delta: 8.02(\mathrm{~d}, J=7.8 \mathrm{~Hz}, 2 \mathrm{H}, \mathrm{Ar}-\mathrm{H})$, 7.50-7.45 (m, 12H, Ar-H), 7.39 (d, $J=7.8 \mathrm{~Hz}, 2 \mathrm{H}, \mathrm{Ar}-\mathrm{H}) ; 7.23(\mathrm{~m}, 2 \mathrm{H}, \mathrm{Ar}-$ $\mathrm{H}), 2.35\left(\mathrm{~s}, 3 \mathrm{H}, \mathrm{CH}_{3}\right)$, IR $(\mathrm{KBr}): 3028(\mathrm{C}-\mathrm{H}), 1644(\mathrm{C}=\mathrm{C}), 1531(\mathrm{Ph}), 1212(\mathrm{Ar}-$ $\mathrm{H}), 890(\mathrm{Ar}-\mathrm{H}) \mathrm{cm}^{-1}$.

1,4,5-triphenyl-2-m-chlorophenylimidazol(4g) white solid, mp: 1644$146{ }^{\circ} \mathrm{C} .{ }^{1} \mathrm{H}$ NMR $(600 \mathrm{MHz}$, DMSO- $d$ ) $\delta:$ 7.57-7.62 (m, 16H, Ar-H), 7.39 (m, 2H,Ar-H); IR (KBr): 3017(C-H), 1650(C=C), 1526(Ph), 1233(Ar-H), 1004(Ar-H), 877(Ar-H), 768(Ar-H) $\mathrm{cm}^{-1}$

\section{SUPPLEMENTS}

Crystallographic data (CCDC-752970 for 3h) have been deposited at the 
Cambridge Crystallographic Database Centre and crystallographic data for the structures can be obtained free of charge from The Cambridge Crystallographic Data Centre via www.ccdc.cam.ac.uk/data_request/cif.

\section{ACKNOWLEDGEMENTS}

This research was supported by the Cultivation and Construction Fund for the State Key Subject of Physical Chemistry. We thank the Testing Center of Yangzhou University for characterizing our compounds.

\section{REFERENCES}

[1] R. W. Brimblecombe, W. A. M. Ducan, G. J. Durant, J. C. Emmett, C. R. Ganellin, M. E. Parons, J. Int. Med. Res. 3, 86, (1975).

[2] Y. Tanigawara, N. Aoyama, T. Kita, K. Shirakawa, F. Komada, M. K. Kasuga, Clin. Pharmacol. Ther. 66, 528, (1999).

[3] W. Hunkaler, H. Mohler, L. Pieri, P. Polc, E. P. Bonetti, R. Cumin, R. W. Schaffner, nature 290, 514, (1981).

[4] P. Wasserscheid, W. Keim, Angew. Chem. Int. Ed. Eng. 39, 37872, (2000).

[5] a) D. E. Frantz, L. Morency, A. Soheili, J. A. Murry, E. J. J. Grabowski, R. D. Tillyer, Orgaic letters. 6(5), 843, (2004).; b) S. E. Wolkenberg, D. D. Wisnoski, W. H. Leister, Y. Wang, Z. Zhao, C. W. Lindsley, Organic Letters. 6(9), 1453, (2004).

[6] S. Balalaie, A. Arabaniam, M. S. Hashtroudi, Mont, Fur. Chem. 131, 945, (2000).
[7] G. V. M. Sharma, Y. Jyothi, P. S. LaKSHMI, Syn. Commun. 36, 2991, (2006).

[8] M. M. Heravi, K. Bakhtiari, H. A. Oskooie, S. Taheri, J. Mol. Catal. A: Chem. 263, 279, (2007).

[9] S. A. Siddiqui, U. C. Narkhede, S. S. Palimkar, T. Daniel, R. J. Lahoti, K. V. Srinivasan, Tetrahedron 61, 3539,. (2005).

[10] M. Kidwai, P. Mothsra, V. Bansal, R. Goyal, Mont. Fur. Chem. 137, 1189, (2006).

[11] G. K. Wagner, D. Kotchenreuther, W. Zimmermann, S. A. Laufer, J. Org. Chem. 68, 4527, (2003).

[12] Y. Fukumoto, K. Savoada, M. Hagihara, N. Chatani, S. Murai, Angew. Chem. Int. 41, 2279, (2002).

[13] S. Laufer, G. Wangner, D. Kotchenreuther, Angew. Chem. Int. 41, 2290, (2002).

[14]L. Nagarapu, S. Apuri, S. Kantevaru, J. of Mol. Cat. A: Chemical. 266, 104, (2007)

[15] M. Kidwai, P. Mothsra, Tetrahedron Letters, 47, 5029, (2006).

[16]X. B. Jing, X. Pan, Z. Li, Y. C. Shi, Syn. Commn. 39(03), 492, (2009).

[17]P. Lidstrom, J. Tierney, B. Wathey, J. Westman, Tetrahedron 57, 9225 , (2001).

[18] B Cui, B. L. Zheng, K. He, Q. Y. Zheng, J. Nat. Prod. 66, 1101, (2003).

[19] a) S. L. Abrahams, R. J. Hazen, A. G. Batson, A. P. J. Phillips, Pharmacol. Exp. Ther. 249, 359, (1989).; b) A. P. Phillips, H. L. White, S. Rosen, Eur. Patent. 58890(A1), (1982). 